\title{
Bioremoval of PVP coated silver nanoparticles using Aspergillus niger. the role of exopolysaccharides
}

Ola M. Gomaa ( $\nabla$ ola_gomaa@hotmail.com )

Egyptian Atomic Energy Authority https://orcid.org/0000-0002-0591-2258

Amar Elrshim

University of South Carolina

Anindya Chanda

University of South Carolina

\section{Research Article}

Keywords: Ag-NPs, Aspergillus niger, bioremoval, exopolysaccharides, Reactive Oxygen Species, Polyvinylpyrrolidone (PVP)

Posted Date: June 14th, 2021

DOI: https://doi.org/10.21203/rs.3.rs-620256/v1

License: (a) (1) This work is licensed under a Creative Commons Attribution 4.0 International License.

Read Full License 


\section{Abstract}

The rapidly growing production and applications of Engineered Nanoparticles (ENPs) foresees a concomitant increase in the exposure of humans to their potential toxic effects through accidental release to the environment. Due to the limited and/or contaminated water resources, the need to re-use treated water has become imperative. The present work aims to study the removal of Polyvinylpyrrolidone coated silver nanoparticles (PVP-Ag-NPs) using Aspergillus niger and depict the role of exopolysaccharides in the removal process. Our results show that the majority of PVP-Ag-NPs were attached to fungal pellets. About $74 \%$ and $88 \%$ PVP-Ag-NPs were removed when incubated with $A$. niger pellets and exopolysaccharide-induced $A$. niger pellets, respectively. lonized Ag decreased by 553 and 1290 fold under the same conditions as compared to stock PVP-Ag-NP. The increase in PVP-Ag-PVP concentrations resulted in an increase in reactive oxygen species (ROS) in $24 \mathrm{~h}$. The UV-Visible spectrum shows the disappearance of $\mathrm{Ag}$ characteristic peak and the broadness of the spectrum suggested an increase in size. Dynamic Light Scattering results showed an increase in PVP-Ag-NPs size from $28.4 \mathrm{~nm}$ to $115.9 \mathrm{~nm}$ for $A$. niger pellets and $160.3 \mathrm{~nm}$ after removal by stress-induced $A$. nigerpellets and further increased to $650.1 \mathrm{~nm}$ for in vitro EPS removal. Our findings show that EPS can be used for nanoparticle removal, by increasing the net size of nanoparticles in aqueous media, this will, in turn, facilitate its filtration through conventional filtration techniques commonly used at wastewater treatment plants.

\section{Introduction}

Engineered nanoparticles (ENPs) are produced in different shapes and forms. They are widely used in gene therapy, drug delivery, agriculture, industry, cosmetics, bioremediation, imaging, and diagnostics (Missaoui et al 2018). In particular, silver nanoparticles (Ag-NPs) are considered one of the most produced nanoparticles due to their different applications, they are used as an anticancer agent (Almalki and Khalifa 2020), anti-inflammatory, antioxidant (Alkhalaf et al 2020), for dye degradation (Ahmad et al 2020), removal of anthropogenic pollutants from water (Sherin et al 2020), for treatment of wastewater (Qu et al 2013), in biosensors (Tran et al 2020), signal amplification (Hou et al 2020) and to enhance thermal energy storage (Pradeep et al 2020). According to EPA fact sheet nanomaterials (2017), 1800 consumer nanomaterial products have reached the market since 2014.

Unfortunately, the enormous development in nanomaterial fabrication and applications has prompted questions about their long-term accumulation in the environment and human surroundings (Radziun et al 2011, Kik et al 2020). Accidental release into soil and sewer systems were reported to affect the viability of soil microflora and activated sludge bacteria which eventually affect their performance (Gomaa 2014), they can easily reach water and drinking facilities (Lawler et al 2013), result in acute toxicity and bioaccumulation of Ag-NPs aquatic organisms (Lacave et al 2017). Different toxicity studies were conducted to evaluate the extent of damage exerted by ENPs (Marimon-Bolívar et al 2019, Liu et al 2019). The toxicity can take place via skin, inhalation, and ingestion and can cause oxidative stress, inflammation, DNA damage, apoptosis, and translocation inside organs and tissues leading to secondary toxicity (Missaoui et al 2018). 
ENPs in general have been reported to be successfully removed via coagulation, flocculation, filtration (Lawler et al 2013), and aggregation (Zhang et al 2020). However, bioremoval using microorganisms and their metabolites is still considered cost-effective, efficient, and environmentally friendly as compared to other chemical and physical treatments (Khan et al 2012, Oh et al 2015). The interaction between microbes and metals, in general, can take place via metabolism-dependent or independent pathways, rendering the metals less toxic or less available to prevent their leaching in the environment magnifying the problem (Gupta and Diwan 2017).

Fungi can remove heavy metals and other pollutants through interaction between metal cations and fungal cell wall functional groups such as carboxylic, hydroxyl, amine, sulfate, and phosphate, groups through complexation, ion exchange, or physical adsorption (Viraraghavan and Srinivasan 2011, Qin et al 2019). Fungi can produce exopolysaccharides (EPS) in the presence of heavy metals as stress response, this produced EPS helps in the exclusion of heavy metals from the media and can be used in heavy metal removal strategies (Mohite et al 2017). Fungal EPS are macromolecular structures with unique conformations, interesting properties for industrial, medical, and environmental applications. Its production is highly dependent on the media composition and physical growth conditions (Mahapatra and Banerjee 2013). EPS can be produced and remain bound to the surface or released in the media, while the latter can be collected, purified, the former provides an additional layer to fungi, thus providing a larger surface area for metal or ENPs adsorption. From this standpoint, the present work aims to provide insight on PVP-Ag-NPs removal from aqueous media using Aspergillus niger, and stress-induced Aspergillus niger and to depict the role of exopolysaccharides in the bioremoval process.

\section{Materials And Methods}

Engineered nanoparticles (ENPs) are produced in different shapes and forms. They are widely used in gene therapy, drug delivery, agriculture, industry, cosmetics, bioremediation, imaging, and diagnostics (Missaoui et al 2018). In particular, silver nanoparticles (Ag-NPs) are considered one of the most produced nanoparticles due to their different applications, they are used as an anticancer agent (Almalki and Khalifa 2020), anti-inflammatory, antioxidant (Alkhalaf et al 2020), for dye degradation (Ahmad et al 2020), removal of anthropogenic pollutants from water (Sherin et al 2020), for treatment of wastewater (Qu et al 2013), in biosensors (Tran et al 2020), signal amplification (Hou et al 2020) and to enhance thermal energy storage (Pradeep et al 2020). According to EPA fact sheet nanomaterials (2017), 1800 consumer nanomaterial products have reached the market since 2014.

Unfortunately, the enormous development in nanomaterial fabrication and applications has prompted questions about their long-term accumulation in the environment and human surroundings (Radziun et al 2011, Kik et al 2020). Accidental release into soil and sewer systems were reported to affect the viability of soil microflora and activated sludge bacteria which eventually affect their performance (Gomaa 2014), they can easily reach water and drinking facilities (Lawler et al 2013), result in acute toxicity and bioaccumulation of Ag-NPs aquatic organisms (Lacave et al 2017). Different toxicity studies were conducted to evaluate the extent of damage exerted by ENPs (Marimon-Bolívar et al 2019, Liu et al 2019). 
The toxicity can take place via skin, inhalation, and ingestion and can cause oxidative stress, inflammation, DNA damage, apoptosis, and translocation inside organs and tissues leading to secondary toxicity (Missaoui et al 2018).

ENPs in general have been reported to be successfully removed via coagulation, flocculation, filtration (Lawler et al 2013), and aggregation (Zhang et al 2020). However, bioremoval using microorganisms and their metabolites is still considered cost-effective, efficient, and environmentally friendly as compared to other chemical and physical treatments (Khan et al 2012, Oh et al 2015). The interaction between microbes and metals, in general, can take place via metabolism-dependent or independent pathways, rendering the metals less toxic or less available to prevent their leaching in the environment magnifying the problem (Gupta and Diwan 2017).

Fungi can remove heavy metals and other pollutants through interaction between metal cations and fungal cell wall functional groups such as carboxylic, hydroxyl, amine, sulfate, and phosphate, groups through complexation, ion exchange, or physical adsorption (Viraraghavan and Srinivasan 2011, Qin et al 2019). Fungi can produce exopolysaccharides (EPS) in the presence of heavy metals as stress response, this produced EPS helps in the exclusion of heavy metals from the media and can be used in heavy metal removal strategies (Mohite et al 2017). Fungal EPS are macromolecular structures with unique conformations, interesting properties for industrial, medical, and environmental applications. Its production is highly dependent on the media composition and physical growth conditions (Mahapatra and Banerjee 2013). EPS can be produced and remain bound to the surface or released in the media, while the latter can be collected, purified, the former provides an additional layer to fungi, thus providing a larger surface area for metal or ENPs adsorption. From this standpoint, the present work aims to provide insight on PVP-Ag-NPs removal from aqueous media using Aspergillus niger, and stress-induced Aspergillus niger and to depict the role of exopolysaccharides in the bioremoval process.

\section{Fungi and growth under exopolysaccharide producing conditions}

Aspergillus niger isolated and characterized in previous work (Gomaa et al 2013). A proper dilution of $A$. niger spore suspension was used to inoculate exopolysaccharide (EPS) producing media $\mathrm{g} / \mathrm{l}$ Glucose 60 , Peptone 20, $\mathrm{MgSO}_{4} .7 \mathrm{H}_{2} \mathrm{O} 2, \mathrm{KH}_{2} \mathrm{PO}_{4} 2, \mathrm{FeSO}_{4} .7 \mathrm{H}_{2} \mathrm{O} 0.05$, pH 5.8 (Li et al 2012), $\mathrm{CaCl}_{2} 1 \mathrm{M}$ was added to the cultivation media on the day of inoculation to induce stress (Gomaa et al 2013) Different glucose concentrations were used $(0,15,30,60$ and $120 \mathrm{~g} / \mathrm{l})$. The flasks were incubated for 4 days at $30^{\circ} \mathrm{C}$ and $150 \mathrm{rpm}$. At the end of incubation, fungal pellets were separated from media using Mira cloth filtration, fungal pellets were weighed after removing excess liquid. The extracellular fluid for each flask was aliquoted in a sterile $50 \mathrm{ml}$ falcon tube, 2 volumes of $95 \%$ ethanol were added to each sample and were left for $48 \mathrm{~h}$ at $4^{\circ} \mathrm{C}$ on a shaker. Samples were centrifuged at $1780 \mathrm{~g}$ for 15 minutes using Thermo Scientific SORVALL LEGENT TR+ centrifuge. The supernatant was discarded and formed pellets were used for EPS analysis. EPS was measured as total carbohydrates using phenol sulfuric method described in Biovision total carbohydrate colorimetric kit user instructions. The absorbance was read at $490 \mathrm{~nm}$ using SpectraMax M3, MultiMode Microplate reader, Molecular Devices, San Jose, CA, USA. For blank 
samples, water was used instead of sample. Concentrations were calculated from a standard curve, glucose was used as the standard. Concavalin A conjugates (Invitrogen detection technologies) Molecular probes were used to label EPS around mycelia. Fluorescence was detected at 555/580 nm using SpectraMax M3, MultiMode Microplate reader, Molecular Devices, San Jose, CA, USA. Images of EPS containing A. niger were captured using Leica model TCS SP5; Leica Microsystems CMS GmbH, Mannheim, Germany.

\section{Engineered nanoparticles (ENPs) understudy}

ENP in this study was Ag- Polyvinyl pyrrolidine (Ag-PVP) Biopure nanospherical particles were purchased from nanocomposix (San Diego, CA, USA), 99.99\% silver purity, Zeta potential -32 mV, diameter (TEM) $20.5 \pm 3.6 \mathrm{~nm}$. Stock suspensions were prepared and sonicated for $30 \mathrm{~s}$ at $50 \mathrm{~W}$ before use.

\section{A. niger growth and Reactive Oxygen Species (ROS) in the presence of PVP-Ag-NPs}

PVP-Ag-NPs were added to $24 \mathrm{~h}$ old $A$. niger pellets in $100 \mathrm{ml}$ Erlenmeyer flasks in the following final concentrations: $0,25,50$, and $100 \mathrm{ppm}$. The flasks were incubated for $24 \mathrm{~h}$ at $30^{\circ} \mathrm{C}$ in a rotatory shaker at $150 \mathrm{rpm}$. Fungal weight was performed as described above. Reactive Oxygen species detection was performed according to Kenne et al (2018). Equal weights $(0.5 \mathrm{~g})$ of fungal pellets of each flask was transferred into 9 well plate. 2',7'-dichlorofluorescein diacetate (DCFH-DA) was used to quantify ROS. For each well, $1 \mathrm{ml}$ Phosphate Buffer Saline (PBS) was added to $1 \mu \mathrm{m}$ DCFD-A, mixed well, and left to incubate at room temperature for $4 \mathrm{~h}$ in dark. Five hundred $\mu \mathrm{l}$ of each sample was transferred to microfuge for a quick spin, $100 \mu \mathrm{l}$ was transferred to 96 well plate. Read at 490/525 nm using SpectraMax M3, MultiMode Microplate reader, Molecular Devices, San Jose, CA, USA.

\section{PVP-Ag-NPs removal by $A$. niger experiments}

To depict the mechanism of PVP-Ag-NPs bioremoval, $24 \mathrm{~h}$ old $A$. niger pellets $(1 \mathrm{~g})$, A. niger culture media containing EPS $(10 \mathrm{ml})$ and EPS induced $A$ nigerpellets $(1 \mathrm{~g})$, were each placed in a separate flask, 50 ppm PVP-Ag-NPs was added and cultures incubated at $150 \mathrm{rpm}, 30^{\circ} \mathrm{C}$ for $24 \mathrm{~h}$. PVP-Ag-NPs concentration and size were assayed as mentioned below.

\section{PVP-Ag-NPs UV-Vis spectrum, concentration and size}

To follow up the bioremoval of PVP-Ag-NPs from the media, Surface Plasmon Resonance (SPR) was used. A dual-beam UV absorbance spectrometer (UV-vis) (Shimadzu UV-2600 spectrophotometer, Co., Kyoto, Japan) at a resolution of $1 \mathrm{~nm}$ from 200-800 nm wavelength range at room temperature. Quartz 
glass cuvettes with an optical path length of $10 \mathrm{~mm}$, requiring. Ultrahigh purity water was used as the reference sample to take the blank spectrum for all measurements. Dynamic light scattering (DLS) was used to determine the particle size distribution (z-average) of the PVP-Ag-NPs suspensions, A. niger, EPS induced $A$. niger and in vitro EPS using a Zetasizer (Nano-ZS, Malvern Instruments Ltd., MA, USA). Inductively coupled plasma-atomic mass spectroscopy (ICP-MS) was used to measure Ag ENPs. The samples were acidified in 68-70\% nitric acid $\left(\mathrm{HNO}_{3}\right)$, trace metal grade, Fisher Scientific, MA, USA) and then diluted 100 fold in $1 \%$ of $\mathrm{HNO}_{3}$, this was followed by analysis via (NexIONTM 350 D, PerkinElmer Inc., Waltham, MA).

\section{Bioremoval and Adsorptive capacity}

To determine the bioremoval and adsorptive capacity of $A$. niger, EPS induced A. niger, and in vitro EPS. Initial and residual PVP-Ag-NPs concentrations were measured as previously described. The removal was calculated by the following equation:

\section{See equation 1 in the supplementary files section.}

where $C_{i}$ and $C_{f}$ are the initial and final lead concentrations, respectively.

While the adsorptive capacity was calculated by the following equation:

\section{See equation 2 in the supplementary files section.}

Where $C_{i}$ is the initial concentration, $C_{f}$ is the final concentration, $W$ is the weight of the immobilized fungal mycelia (adsorbent) in $\mathrm{gm}, \mathrm{V}$ is the volume of the sample. All the data presented are the mean value of three readings $\pm \mathrm{SE}$.

\section{Results}

\section{Exopolysaccharide production using Aspergillus niger}

The increase of glucose concentration to A. niger growth media has led to an increase in both released and surface-bound EPS production. Fig. 1a shows that EPS released in the media increased from 12.05 to $34.2 \mathrm{mg} / \mathrm{ml}$ as concentrations increased from 0 to $120 \mathrm{~g} / \mathrm{l}$, respectively, on the other hand, surfacebound glucose increased from 10.8 to $46.07 \mathrm{mg} / \mathrm{ml}$, respectively. Despite the increase in EPS production, yet the fungal growth reached its peak weight of $18.3 \mathrm{~g} / 100 \mathrm{ml}$ at $60 \mathrm{~g} / \mathrm{l}$ glucose concentration, but dropped to $14 \mathrm{~g} / 100 \mathrm{ml}$ at $120 \mathrm{~g} / \mathrm{l}$. For the upcoming experiments, $60 \mathrm{~g} / \mathrm{l}$ glucose was used for media preparation. To increase EPS production, calcium chloride was added to the media on the day of inoculation. Fig. 1b shows that EPS released in the media increased from 16.6 to $19.7 \mathrm{mg} / \mathrm{ml}$ in the absence and presence of calcium chloride, respectively, while surface-bound EPS increased from 25.7 to $34.3 \mathrm{mg} / \mathrm{ml}$ in the absence and presence of calcium chloride, respectively. On the other hand, the presence or absence of calcium chloride did not affect fungal growth. Fig. $1 \mathrm{c}$ represents the appearance of EPS as fluorescent spots in A. niger grown in the absence and calcium chloride-containing media. 


\section{PVP-Ag-NP removal by Aspergillus niger pellet and growth media}

A.niger pellets removed about $74 \%$ of PVP-Ag-NPs, while $26 \%$ was removed by $A$. niger growth media (Fig. 2a). The UV-Vis spectrum reveals the disappearance of the Ag distinct peak at $392-400 \mathrm{~nm}$, the overall absorbance increased which reflects the presence of organic macromolecules (Fig. 2b).

\section{Aspergillus niger stress response to Ag-PVP nanoparticles}

The exposure of Aspergillus niger to PVP-Ag-NPs has resulted in an increase in reactive oxygen species (ROS) of about 4.41 fold in intensity. The increase was directly proportional to the concentration of nanoparticles present in the media (Fig. 3). Although the fungus showed ROS upon exposure to the used nanoparticles, yet the fungal growth was not compromised.

\section{PVP-Ag-NPs bioremoval using Aspergillus niger}

Fig. 4a shows the removal of PVP-Ag-NP increased when using EPS induced $A$. niger pellets. The ionized Ag decreased 552 and 1290 fold after removal using A. nigerpellets and EPS induced A. nigerpellets, respectively. Fig. $4 \mathrm{~b}$ shows the UV-Vis spectrum before and after ENPs removal, the peak at $400 \mathrm{~nm}$ distinct for Ag disappeared after using $A$. nigerpellets and the overall absorbance increased.

Table 1 represents the change in sizes for the different samples the size of Ag-PVP-NPs has increased from $28.8 \mathrm{~nm}$ to $115.9 \mathrm{~nm}$ when it was removed by $A$. niger pellets, $160.3 \mathrm{~nm}$ after removal using EPS induced A. niger pellets, and $650.1 \mathrm{~nm}$ when A. niger growth media containing $100 \mathrm{mg} / \mathrm{ml}$ EPS was used for bioremoval. The adsorptive capacity of the different samples increased from 1.28 to $2.85 \mathrm{mg} / \mathrm{g}$ and jumped to $3.45 \mathrm{mg} / \mathrm{g}$ when EPS (100 mg/ml) from media was used

\section{Discussion}

Exopolysaccharides are known to be adhesive substances that can adsorb different pollutants. Both media composition and physical conditions play an integral role in the production and composition of EPS by fungi (Mahapatra and Banerjee 2013). While it is commonly reported that glucose, peptone, and Magnesium sulfate are the key elements in EPS production by fungi (Li et al 2012, Sharmila et al 2017), yet metal stress was also reported to induce EPS production as means of protection (Mohite et al 2017). In previous work, calcium chloride was reported to induce stress in A. niger (Gomaa et al 2013). Calcium chloride was also reported to be among media components inducing EPS production in fungi (Mahapatra and Banarjee 2013). Therefore, we used calcium chloride to induce stress and therefore induce EPS production in A. niger. EPS produced in our study was evident as fluorescent spots on the surface of $A$. 
niger mycelial pellets. The fluorescent spots reflected the intensity of the mannose and glucose binding conjugate dye Concavalin A used in the present study.

EPS produced from bacterial, algal, and fungal origin are recommended as surface-active agents for heavy metal removal via adsorption of heavy metal cations onto the negative charge of EPS functional groups, this process is metabolism-independent (Rasulov et al 2013). PVP-Ag-NPs have a total surface charge of $-32 \mathrm{mV}$ (according to the manufacture's certificate of analysis), this suggests that removal of the NPs will not depend on physicochemical adsorption. However, testing ROS produced in presence of NPs, ROS was found to increase upon the increase in NPs concentration in the media. On the other hand, fungal growth was not affected by the presence of the tested NP concentrations. This result is an indication that sublethal doses of nanoparticles are still a threat and can cause stress to living cells. This result is in accordance with Meyer et al (2010) and Zhang et al (2015) who reported concomitant toxicity of Ag and ZnO nanoparticles using Caenerhabitis elegans and Schoenoplectus tabernaemontani, respectively. PVP coating of nanoparticles is performed to avoid agglomeration, decrease particle size and ensure uniform dispersion (Gharibshahi et al 2017). The small size of PVP-coated nanoparticles and their stability in solutions make them more difficult to remove. However, ROS production was reported to cause the removal of PVP coat from PVP-Ag-NPs (Zhang et al 2020), thus exposing Ag divalent cation. This in turn can result in adsorption of $\mathrm{Ag}^{++}$to the negatively charged EPS or negatively charged fungal cell wall (Gupta and Diwan 2017).

From our perspective, both fungi and their produced EPS represent a low-cost, highly efficient adhesive material that can remove PVP-Ag-NPs from aqueous media. The macromolecular structure and net negative charge of both fungi (Viraraghavan and Srinisivan 2011) and EPS provide a suitable matrix for entrapment of pollutants and thus it is used as flocculants (Sharmila et al 2014) in addition to its use as a bioadsorbent for heavy metals (Gupta and Diwan 2017). Based on its nature, it was expected to remove ENPs from aqueous media. In the present study, EPS-induced $A$. niger pellets removed the majority of PVP-Ag-NPs from aqueous media as opposed to a smaller fraction when using EPS-containing media.

It is clear from our results that UV-Visible spectrum broadness and intensity increased after PVP-Ag-NPs removal using $A$. niger, EPS induced $A$. niger, media containing. Baset et al (2011) reported that the width of the UV-Visible profile has been related to NP size, while the intensity of absorption spectra is related to the dielectric constant and interband transition of metal nanoparticles. The present study also shows that PVP-Ag-NPs size has increased after removal by A. niger, EPS induced A. niger, media, and in vitro EPS. The diameter of particles contributes to the aggregation and flocculation of Ag-NPs (Oh et al 2015, Zhang et al 2020). The increase in size suggests that bioremoval took place and is relevant to the adsorptive capacity of the used adsorbent in the present study. The obtained results suggests also that in vitro EPS can be used for NP removal and this will be our focus in the future.

Based on the current findings we can confirm that $A$. niger is responsible for the majority of PVP-Ag-NPs removal and EPS containing media contributes but to a small extent. We can depict that the removal takes place via one of the three following mechanisms; 1) EPS bound to A. niger pellets is responsible for 
removal, by providing more surface area, 2) EPS can be induced by exposing $A$. niger to stress or 3) by increasing the size of PVP-Ag-NPs, through coating by EPS or binding to it, or through aggregation (Fig. $5)$.

\section{Conclusion}

The present study concludes that $A$. niger can efficiently remove PVP-engineered nanosilver particles. The bioremoval process can take place via different mechanisms with EPS playing an integral role. Whether through entrapment, adsorption, or aggregation, the result can be implemented in wastewater plants prior to a filtration step in contained vessels. EPS is easily produced and can be used in partially purified form, it can be combined with other biomaterials to provide an efficient biofilter with enhanced mechanical properties. Moreover, it is biodegradable thus considered ecofriendly and cheap. Our future work will focus on studying EPS expression and up-regulation under low-cost media, in addition to testing its use for the removal of different types of NPs under different environmental conditions. We also aim to fabricate an EPS-based biofilter to be used in wastewater treatment tanks.

\section{Declaration}

\section{Conflict of Interest}

The authors declare that the research was conducted in the absence of any commercial or financial relationships that could be construed as a potential conflict of interest.

\section{Funding}

This work was part of Fulbright 2018/2019 fellowship awarded to Ola M. Gomaa to Department of Health Sciences, Arnold School of Public Health, University of South Carolina, SC, USA.

\section{References}

Ahmad, T., Nazim, A. V., Farooq, U., Khan, H., Sapan, K., Ubaidullah, M. J., Ahmed, J. (2020). Biosynthesis, characterization and photo-catalytic degradation of methylene blue using silver nanoparticles. Materials Today: Proceedings (in press).

Alkhalaf, M., Hussein, R., Hamza, A. (2020). Green synthesis of silver nanoparticles by Nigella sativa extract alleviates diabetic neuropathy through anti-inflammatory and antioxidant effects. Saudi J. Biol. Sci. (in press). 
Almalkia, M., Khalifaa, A. (2020). Silver nanoparticles synthesis from Bacillus sp KFU36 and its anticancer effect in breast cancer MCF-7 cells via induction of apoptotic mechanism

J. Photochem. \& Photobiol., B: Biol. 204, 111786.

Baset, S., Akbari, H., Zeynali, H., Shafie, M. (2011). Size measurement of metal and semiconductor nanoparticles via UV-Vis absorption spectra. Digest J Nanomaterials and Biostructures. 6, 709-716.

Environmental Protection Agency Fact Sheet (2017). https://www.epa.gov/sites/production/files/201403/documents/ffrrofactsheet_emergingcontaminant_nanomaterials_jan2014_final.pdf

Gharibshahi, L, Saion, E, Gharibshahi, E., Shaari, A.H., Matori, K.A. (2017) Influence of Poly

(vinylpyrrolidone) concentration on properties of silver nanoparticles manufactured by modified thermal treatment method. PLoS ONE 12(10):e0186094. https://doi.org/10.1371/journal.pone.0186094

Gomaa,O. (2014). Toxicity removal of silver nanoparticles by live and heat shock Aspergillus niger. World J. Microbiol. Biotechnol. 30,1747-1754.

Gomaa, O., Selim, S., Linz,J (2013). Biochemical and biophysical response to calcium chloride stress in Aspergillus niger and its role in malachite green degradation. Cell Biochem. Biophys. 65, 413-423.

Gupta, P., Diwan, B. (2017). Bacterial Exopolysaccharide mediated heavy metal removal: A Review on biosynthesis, mechanism and remediation strategies. Biotechnol. Reports 13, 58-71.

Hou, L., Huang, Y., Hou, W., Yan, Y., Liu, J., Xia, N.(2020). Modification-free amperometric biosensor for the detection of wild-type p53 protein based on the in situ formation of silver nanoparticle networks for signal amplification. Int. J. Biol. Macromol. 158, 580-586

Kenne, G., Gummadidala, P., Omebeyinje, M., Mondal, A. K., Bett, D., McFadden, S., Bromfield, S., Banaszek, N., Velez-Martinez, M., Mitra, C., Mikell, I., Chatterjee, S., Wee, J., Chanda, A. (2018). Activation of Aflatoxin Biosynthesis Alleviates Total ROS in Aspergillus parasiticus

Toxins. 10, 57; doi:10.3390/toxins10020057

Page $10 / 18$ 
Khan, S.S., Mukherjee, A., Chandrasekaran, N. (2012). Adsorptive removal of silver nanoparticles (SNPs) from aqueous solution by Aeromonas punctate and its adsorption isotherm and kinetics. Colloids Surf. B 92, 156-160.

Kik, K., Bukowska, B., Sicinska, P. (2020). Polystyrene nanoparticles: Sources, occurrence in the environment, distribution in tissues, accumulation and toxicity to various organisms. Environ. Pollution. $262,114297$.

Lacave, J.M., Fanjul, A., Bilbao, E., Gutierrez, N., Barrio, I., Arostegui, I., Cajaraville, M., Orbea, A. (2017). Acute toxicity, bioaccumulation and effects of dietary transfer of silver from brine shrimp exposed to PVP/PEl-coated silver nanoparticles to zebrafish Comparat. Biochem. Physiol., Part C 199, 69-80.

Lawler, D. F., Mikelonis, A. M., Kim, I., Lau, B. L., \& Youn, S. (2013). Silver nanoparticle removal from drinking water: Flocculation/sedimentation or filtration? Water Sci \& Technol: Water Supply. 13, 11811187. doi: $10.2166 /$ ws.2013.125.

Li, P., Xu, L., Mou, Y., Shan, T., Mao, Z.. Lu, S., Peng, Y., Zhou, L. (2012). Medium Optimization for Exopolysaccharide Production in Liquid Culture of Endophytic Fungus Berkleasmium sp. Dzf12

Int. J. Mol. Sci. 13, 11411-11426; doi:10.3390/ijms130911411.

Liu, C., Chiu, H., Sung, H., Yeh, J., Wud, K., Liu, S. (2019). Acute oral toxicity and repeated dose 28-day oral toxicity studies of MIL-101Nanoparticles. Regulat. Toxicol. Pharmacol. 107, 104426.

Marimon-Bolívara, W., Tejeda-Benítez, L., Núñez-Avilés, C., Léon-Péreza, D. (2019). Evaluation of the in vivo toxicity of green magnetic nanoparticles using Caenorhabditis elegans as a biological model. Environ. Nanotechnol., Monitoring \& Manag. 12, 100253.

Mahapatra, S., Banerjee, D. (2013). Fungal Exopolysaccharide: Production, Composition and Applications. Microbiol. Insights. 6: 1-16. 
Missaouia, W., Arnold, R., Cummings, B. (2018). Toxicological status of nanoparticles: What we know and what we don't Know. Chemico-Biological Interact. 295, 1-12.

Mohite, B.V., Koli, S.H., Narkhede, C.P., Patil, S.N., Patil, S.V. (2017). Prospective of Microbial Exopolysaccharide for Heavy Metal Exclusion. Appl. Biochem. Biotechnol. 183, 582-600.

Oh, S., Sung, H., Park, C., Kim, Y. (2015). Biosorptive removal of bare-, citrate-, and PVP-coated silver nanoparticles from aqueous solution by activated sludge. J. Industrial Eng. Chem. 25, 51-55

Pradeep, N., Paramasivam, K., Rajesh, T., Subash Purusothamanan, V., Iyahraja, S. (2020). Silver nanoparticles for enhanced thermal energy storage of phase change materials. Mat. Today: Proceedings (in press).

Qin, H., Hu, T., Zhai, Y., Lu, N., Aliyeva, J. (2019). The improved methods of heavy metals removal by biosorbents: A review. Environment. Pollution.113777.

Qu, X., Alvarez, P., Li, Q. (2013). Applications of nanotechnology in water and wastewater treatment. Water Res. 47, 3931-3946.

Radziun, E., Wilczyn'ska, D., Ksiazek, I., Nowak, K., Anuszewska, E.L.. Kunicki, A., Olszyna, A., Zabkowski, T. (2011). Assessment of the cytotoxicity of aluminium oxide nanoparticles on selected mammalian cells. Toxicol In Vitro 25, 1694-1700.

Rasulov, B., Yili, A., Aisa, H. (2013). Biosorption of Metal lons by Exopolysaccharide Produced by Azotobacter chroococcum XU1 J. Environ. Protection. 4, 989-993.

Sharmila, K., Alagarsamy, K., Thillaimaharani, Durairaj, R., Kalaiselvam, M. (2014). Production and characterization of exopolysaccharides (EPS) from mangrove filamentous fungus, Syncephalastrum sp. African J. Microbiol. Res. 8, 2155-2161.

DOI: 10.5897/AJMR12.2341

Page $12 / 18$ 
Sherin, L., Sohail, A., Amjad, U., Mustafa, M., Jabeend, R., Ul-Hamide, A. (2020). Facile green synthesis of silver nanoparticles using Terminalia bellerica kernel extract for catalytic reduction of anthropogenic water pollutants. Colloid and Interface Sci. Comm. 37, 100276.

Tran, H., Nguyen, N., Tran, C., Tran, L., Le, T., Tran, H., Piro, B., Huynh, C., Nguyen, T., Nguyen, N., Hue Dang, T., Nguyen, H., Tran, L., Phan, N. (2020). Silver nanoparticles-decorated reduced graphene oxide: A novel peroxidase-like activity nanomaterial for development of a colorimetric glucose biosensor Arabian J. Chem. (in press).

Viraraghavan, T., Srinivasan, A. (2011). Fungal biosorption and biosorbents. Microbial biosorption of metals. Springer. p. 143-158.

Zhang, Z., Wu, Q., Shang, E., Wang, X.,Wang, K., Zhao, J., Duan, J., Liu, Y., Li, Y. (2020). Aggregation kinetics and mechanisms of silver nanoparticles in simulated pollution water under UV light irradiation. Water Environ. Res. 92, 840-849.

\section{Table}

Table 1: Size $(\mathrm{nm})$ and adsorptive capacity $\left(\mathrm{Q}_{\mathrm{t}}\right)$ of different samples after bioremoval

\begin{tabular}{|lll|}
\hline Sample & Size $(\mathrm{nm})$ & Adsorptive capacity $\left(\mathrm{Q}_{t}\right) \mathrm{mg} / \mathrm{g}$ \\
\hline PVP-Ag-NP & 28.43 & - \\
\hline A.niger & 115.9 & $1.28 \pm 0.01$ \\
\hline EPS induced A. niger & 160.3 & $1.85 \pm 0.01$ \\
\hline EPS $(100 \mathrm{mg} / \mathrm{ml})$ in media & 650.1 & $3.35 \pm 0.08$ \\
\hline
\end{tabular}

\section{Figures}



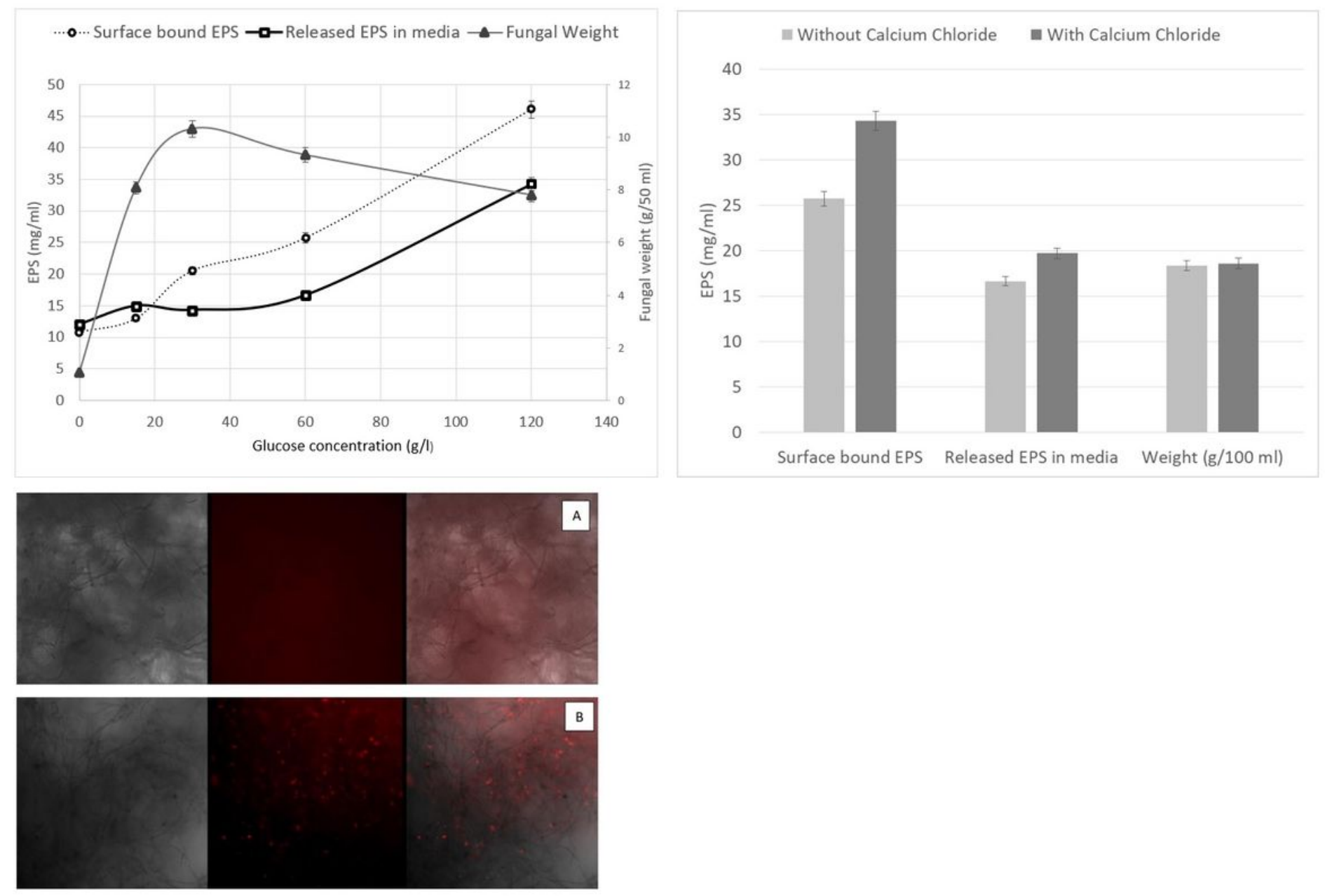

\section{Figure 1}

Fig. 1a: Effect of increasing glucose concentration on surface bound EPS, released EPS and fungal weight after incubation at $30 \mathrm{oC}$ at $150 \mathrm{rpm}$ for 4 days. Fig. 1b: Released and surface bound EPS production and fungal growth in presence and absence of calcium chloride. Fig. 1c: Images of A. niger grown in the presence (B) and absence of calcium chloride (A). Images captured using fluorescent microscope, concavalin A was used to stain EPS 


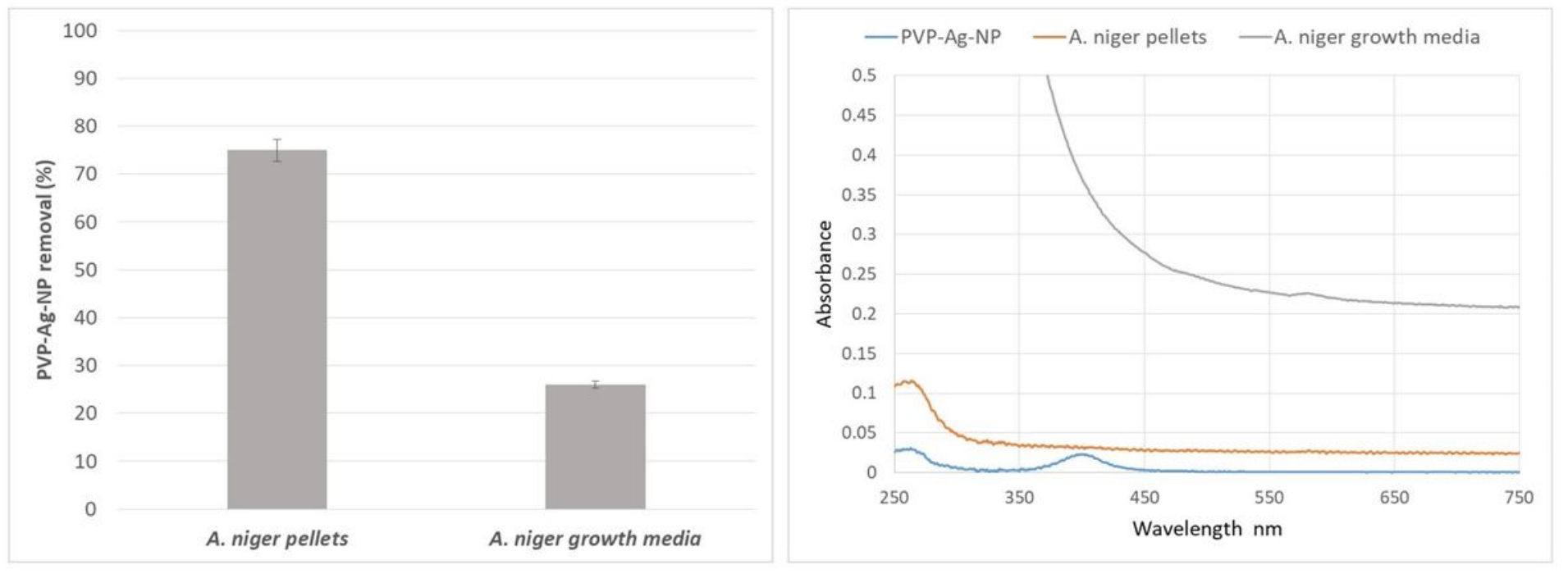

\section{Figure 2}

Fig. 2a: Bioremoval of PVP-Ag-NP using fungal pellets and fungal media containing released EPS. Fig. 2b: UV-Vis spectrum of PVP-Ag-NP before and after bioremoval using A. niger pellets and A. niger growth media containing released EPS. 


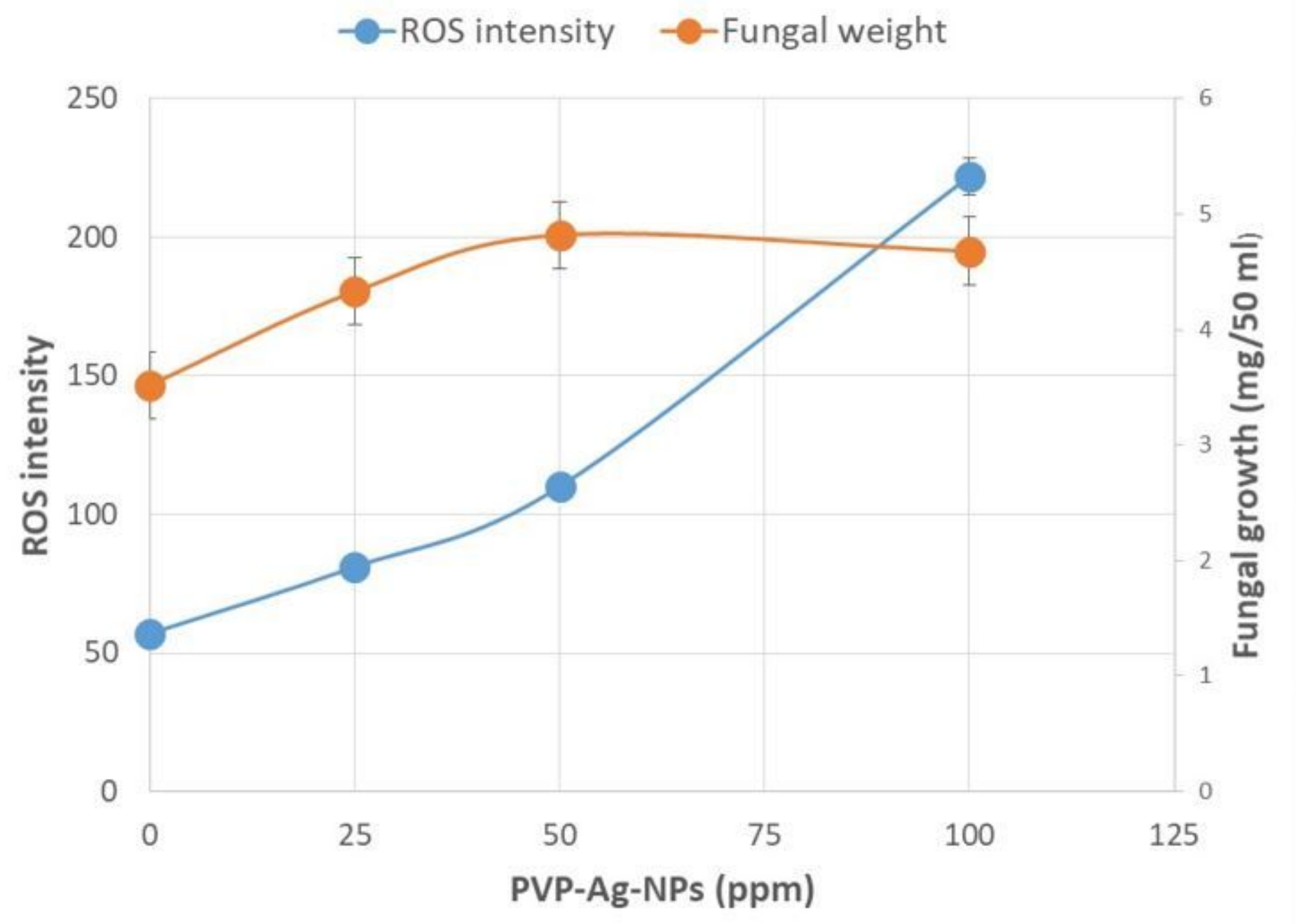

Figure 3

Fig.3: Effect of adding different PVP-Ag-NPs concentrations on ROS and fungal weight of A. niger after $24 \mathrm{~h}$ incubation.
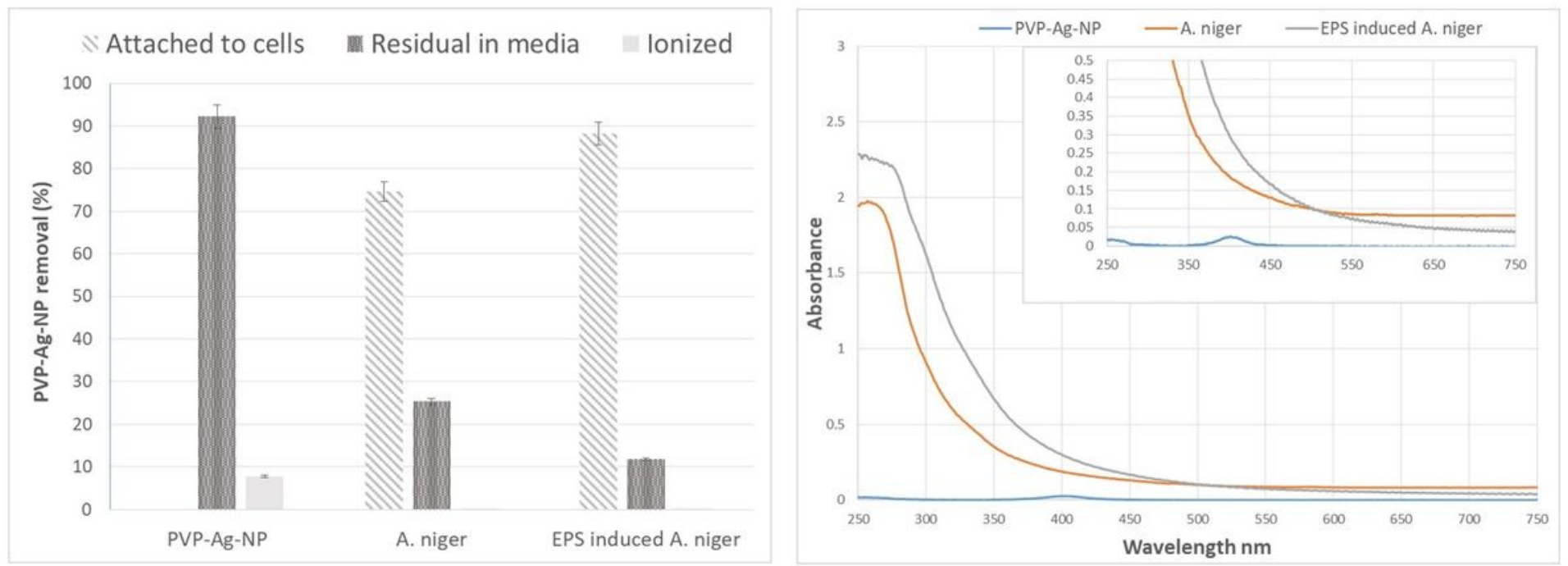


\section{Figure 4}

Fig 4a. Residual, attached and ionized PVP-Ag-NP (\%) after $24 \mathrm{~h}$ incubation with A. niger and EPS induced A. niger pellets as compared to stock Ag-PVP-NP. Fig. 4b: UV-Vis spectrum of PVP-Ag-NP before and after bioremoval using A. niger pellets and EPS induced A. niger pellets.

1)

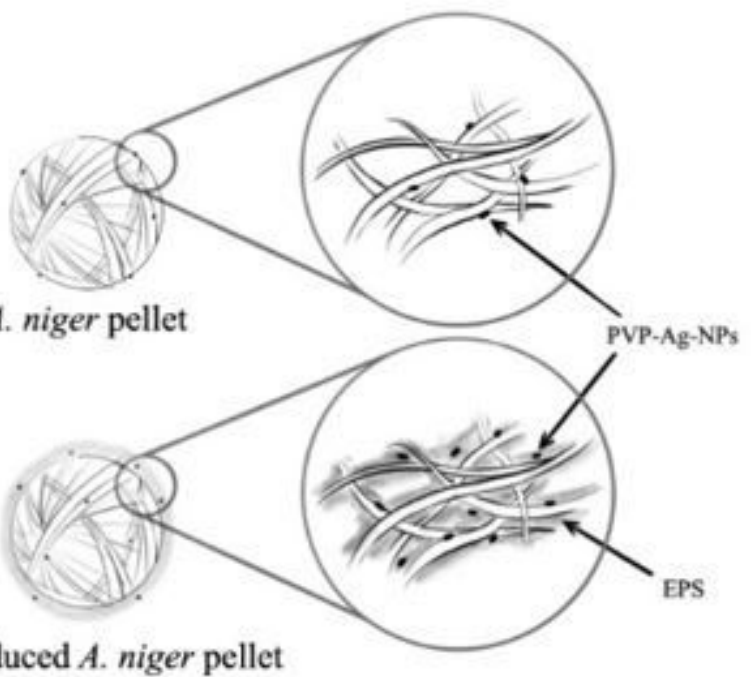

EPS induced $A$. niger pellet

2)

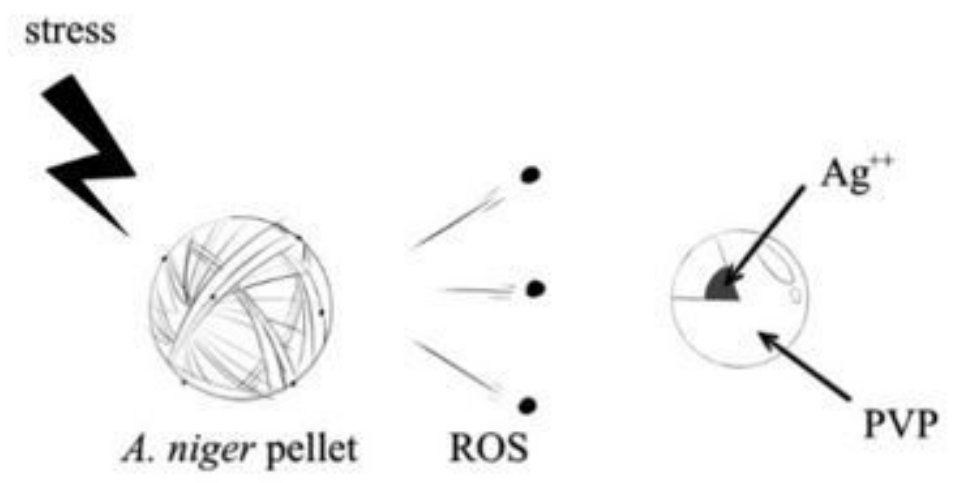

3)
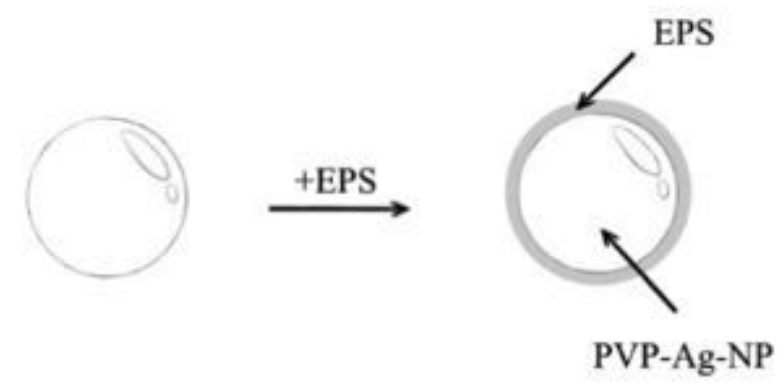

\section{Figure 5}

Fig. 5: Possible mechanisms of PVP-Ag-NP bioremoval using A. niger, EPS induced A. niger and EPS. 


\section{Supplementary Files}

This is a list of supplementary files associated with this preprint. Click to download.

- equations.docx 